\title{
Check-list de Picramniales e Sapindales (exceto Sapindaceae) do estado de Mato Grosso do Sul
}

\author{
José Rubens Pirani \& Cíntia Luíza da Silva-Luz
}

Universidade de São Paulo. Instituto de Biociências, Rua do Matão 277, Cidade Universitária, CEP 05508-090, São Paulo, SP. pirani@usp.br

Recebido em 27.IX.2014

Aceito em 06.IX.2016

DOI 10.21826/2446-8231201873s301

RESUMO - As listas de espécies de Picramniales e Sapindales (exceto Sapindaceae) ocorrentes no Mato Grosso do Sul foram compiladas com base em monografias, floras e revisões taxonômicas publicadas, e dados da Lista de Espécies do Brasil e dos acervos de vários herbários. Os seguintes números de espécies foram reportados em cada família: Picramniaceae (duas spp.), Anacardiaceae (15 spp.); Burseraceae (quatro spp.), Meliaceae (15 spp.), Rutaceae (22 spp.), Simaroubaceae (cinco spp.). Cada táxon é acompanhado de citação de um voucher e dos domínios e habitats em que ocorre no Mato Grosso do Sul.

Palavras-chave: Anacardiaceae, Burseraceae, Meliaceae, Rutaceae, Simaroubaceae.

ABSTRACT - Checklist of Picramniales and Sapindales (excluding Sapindaceae) from the state of Mato Grosso do Sul. This compilation of species of Picramniales and Sapindales (except Sapindaceae) occurring in Mato Grosso do Sul, Brazil, is based on data from monographs, floras and taxonomic revisions, from the Lista de Espécies do Brasil, and from several herbaria. The following numbers of species were reported for each family: Picramniaceae (two spp.), Anacardiaceae (15 spp.), Burseraceae (four spp.), Meliaceae (15 spp.), Rutaceae (22 spp.) Simaroubaceae (five spp.). Each species name is followed by a voucher citation and by information of the domains and habitats in which they occur in Mato Grosso do Sul.

Keywords: Anacardiaceae, Burseraceae, Meliaceae, Rutaceae, Simaroubaceae.

\section{INTRODUÇÃO}

A ordem Picramniales inclui apenas a pequena família neotropical Picramniaceae. Nas filogenias atuais essa ordem emerge como grupo-irmão do clado que contém a ordem Sapindales e outras três ordens, todas inseridas na superodem Rosanae (APG-III 2009, Chase \& Reveal 2009).

Picramniaceae, família erigida em 1995, é composta por três gêneros, dois deles eram tradicionalmente inseridos na família Simaroubaceae e um foi descrito recentemente. Tem cerca de 46 espécies, exclusivamente neotropicais. São plantas lenhosas com folhas compostas pinadas, flores diminutas em pequenos glomérulos dispostos em racemos, espigas ou tirsos ramosos, com flores portando estames em número igual ao de pétalas (três a cinco), e ovário constituído por dois a três carpelos unidos originando um fruto baga ou cápsula alada. As flores são unissexuadas e as plantas dioicas (femininas ou masculinas). A família está representada no Brasil por uma espécie amazônica do gênero Alvaradora, e pelo gênero Picramnia, com 21 espécies, sendo 12 delas restritas ao país (endêmicas), com diversidade concentrada na Amazônia e na Mata Atlântica. A maioria das espécies de Picramnia do Brasil são arvoretas do interior de florestas úmidas, frequentemente conhecidas como cafezinho-do-mato ou café-de-bugre, devido aos frutos carnosos avermelhados a enegrecidos, muito apreciados por aves.

A ordem Sapindales compreende nove famílias. São plantas lenhosas geralmente com folhas compostas, flores dialipétalas uni ou bissexuadas, com disco nectarífero evidente e um notável potencial secretor: látex, resinas, óleos são amplamente encontrados no grupo. Sapindales está representada no Brasil por seis famílias, cinco delas tratadas neste capítulo.

Anacardiaceae é uma família constituída por 81 gêneros e cerca de 800 espécies essencialmente tropicais, e representada no Brasil por 14 gêneros e 55 espécies, sendo 16 delas restritas ao país (Silva-Luz \& Pirani 2017). Trata-se de um grupo de plantas lenhosas resiníferas com folhas simples ou compostas, flores pouco vistosas e frutos carnosos ou secos. No Brasil a diversidade está concentrada nas regiões Norte e Sul, sendo Anacardium e Thyrsodium os gêneros com maior riqueza específica no norte, enquanto Schinus é o principal representante no sul. No centrooeste, nordeste e sudeste, com exceção de algumas poucas espécies endêmicas de cada região, predominam táxons amplamente distribuídos nos domínios do Cerrado e da Mata Atlântica e menos frequentemente na Caatinga.

Burseraceae está constituída por 18 gêneros com cerca de 540 espécies, principalmente pantropicais. São árvores 
e arbustos resiníferos (triterpenóides e óleos voláteis, com característico odor de manga verde percebido ao se amassar folhas ou remover parte da casca). As folhas são geralmente imparipinadas e os folíolos opostos frequentemente com pulvinos desenvolvidos. As flores são pequenas e unissexuadas (em plantas dioicas ou poligâmicas), produzidas em inflorescências condensadas axilares, e os frutos são drupas deiscentes resiníferas. No Brasil ocorrem 104 espécies, 21 delas restritas ao país (Daly \& Perdiz 2017).

Meliaceae é uma família de cerca de 50 gêneros e 550 espécies, essencialmente pantropical. Caracteriza-se pelas folhas pinadas e pelas flores com tubo estaminal, unissexuadas ou bissexuadas. No Brasil estão documentadas 88 espécies, 32 delas endêmicas do país (Flores, 2017). Habitam principalmente as florestas ombrófilas, mas podem estar bem representadas em matas semideciduais e deciduais e bem esporadicamente em áreas abertas de campo e cerrado. Os gêneros mais diversificados no Brasil são Guarea e Trichilia, enquanto os cedros (Cedrela spp.) têm poucas espécies porém muito comuns na maioria das florestas primárias do país e com amplo uso na indústria madeireira e reflorestamentos.

Rutaceae consta de cerca 1900 espécies distribuídas em 160 gêneros, ocorrendo em maior abundância pelas regiões tropicais e subtropicais do mundo todo, principalmente na América tropical, sul da África e Austrália. A característica mais marcante dessa família é a presença de pontuações translúcidas nas folhas e em quase todos os órgãos, correspondentes a cavidades glandulares multicelulares produtoras de óleos voláteis aromáticos. A família é representada no Brasil por 32 gêneros, cinco deles endêmicos, e 195 espécies, sendo 107 delas restritas ao país. No Brasil a diversidade está concentrada na Amazônia e na Mata Atlântica, sendo os gêneros com maior riqueza específica: Conchocarpus (48 espécies no Brasil, 37 endêmicas) e Zanthoxylum (26 espécies no país, 8 endêmicas) (Pirani et al., 2017a). As Rutáceas brasileiras são plantas lenhosas que habitam predominantemente as florestas úmidas, porém algumas (como Esenbeckia grandiflora Mart.) podem constar como as espécies de maior abundância em muitas florestas estacionais semideciduais. Ocorrem geralmente na submata, mas podem também ser árvores de grande porte e fornecedoras de madeiras comercialmente úteis. Alguns dos gêneros bem diversificados em florestas apresentam uma ou algumas espécies habitando formações abertas ou secas (cerrados e campos). Zanthoxylum rhoifolium Lam. é uma espécie muito comum e das mais amplamente distribuídas em todo território brasileiro, ocorrendo em várias formações vegetacionais, incluindo formações secundárias, em contraste com numerosas espécies restritas a habitats específicos ou a territórios pouco extensos, sendo que 28 espécies estão catalogadas como raras pela Conservação Internacional.

Simaroubaceae, na sua circunscrição atual, abrange 22 gêneros e cerca de 109 espécies, de ocorrência principalmente em regiões tropicais e subtropicais do mundo. Apenas oito gêneros ocorrem no Novo Mundo. São plantas lenhosas com folhas compostas pinadas, flores com estames dotados de apêndice evidente na base e gineceu de dois a cinco carpelos unidos apenas pelos estiletes, que originam frutículos samaroides ou drupáceos, unisseminados. Podem ser hermafroditas (Simaba) ou mais frequentemente dioicas. Produzem triterpenoides do tipo quassinoides, conhecidos exclusivamente na família. Está representada no Brasil por seis gêneros e 30 espécies, sendo 14 delas restritas ao país (endêmicas), com diversidade concentrada na Amazônia e na Mata Atlântica (Pirani et al. 2017b). O principal uso de simaroubáceas no Brasil é a extração da madeira marupá (Simarouba amara Aubl.) na Amazônia. Muitas espécies de Simaba são arbustos conhecidos com o sugestivo nome de paratudo e reputadas como medicinais.

\section{Principais acervos}

O principal acervo com material representativo de Sapindales do Mato Grosso do Sul no estado é o herbário da Universidade Federal (CGMS) em Campo Grande. Outros quatro herbários no estado estão listados na Rede Brasileira de Herbários em Dourados, em Corumbá, em Três Lagoas e o da EMBRAPA em Campo Grande. Porém coletas do grupo provenientes do Mato Grosso do Sul são mais numerosas e mais facilmente acessíveis em outros grandes herbários do país, como MBM, R, RB, SP, SPF, UB e UEC.

\section{Principais Grupos de pesquisa}

Os atuais especialistas em Rutaceae do Brasil são Jacquelyn Kallunki (New York Botanical Garden), Milton Groppo (FFCLRP-USP), Pedro Dias (EACH-USP) e José R. Pirani (IB-USP), todos atuando em colaborações variadas; em Picramniaceae e Simaroubaceae atuam Wayt Thomas (NYBG) e José R. Pirani (USP), que têm colaborado há vários anos. $\mathrm{O}$ maior especialista em Burseraceae neotropicais é Douglas Daly (NYBG) e em Meliaceae Terence D. Pennington (RBG-Kew). Em Anacardiaceae, destacam-se as atuações de John Mitchell (NYBG) e Susan Pell (Brooklyn Botanical Garden) nos EUA e, no Brasil, de Cíntia Luiza Silva-Luz, que fez um mestrado sobre o grupo e desenvolve tese de doutorado com Schinus no IBUSP, sob orientação de J.R. Pirani.

\section{Principais lacunas do conhecimento}

Aparentemente há muitas lacunas de coleta de Picramniaceae e das famílias de Sapindales no Mato Grosso do Sul, e a perda de habitats pelas atividades antrópicas parece estar levando a diminuição das populações de plantas desses grupos, sobretudo nos casos das várias espécies de distribuição restrita e que normalmente formam populações pouco numerosas. No caso das Simaba conhecidas pelo sugestivo nome de paratudo e reputadas como medicinais, o extrativismo pelas comunidades tradicionais dessas espécies já originalmente 
pouco abundantes na natureza pode ter contribuído para a situação de raridade extrema de algumas delas, como S. warmingiana Engl. (de ocorrência esperada no MS por estar presente nos cerrados da área de Rondonópolis, no sul do MT) ou S. salubris Engl., documentada no estado por apenas uma coleta da primeira metade do século passado. Muitas árvores de Sapindales têm um histórico de exploração predatória intensiva, sobretudo das espécies fornecedoras de madeira de boa qualidade, como o gonçalo-alves (Astronium fraxinifolium), o guaritá (Astronium graveolens), a braúna (Schinopsis brasiliensis) e o pau-marfim (Balfourodendron riedelianum).

\section{MATERIAL E MÉTODOS}

A compilação desta lista de espécies baseou-se nas monografias clássicas da Flora Brasiliensis (Engler 1874a,b, 1876), nas listas organizadas por Dubs (1998), na sinopse de Picramnia do Brasil (Pirani 1990), nas monografias de Meliaceae (Pennington 1981), de Cedrela (Pennington \& Muellner 2010), de Schinus (Barkley 1957), de Lithraea (Barkley 1962), de Astronium (Barkley 1968) e da subtribo Pilocarpinae (Kaastra 1982), na revisão de Myracrodruon (Santin \& Leitão-Filho 1991), na revisão de Hortia (Groppo \& Pirani 2012), na revisão de Balfourodendron e Helietta (Pirani 1998), na sinopse de Rutaceae do Brasil (Pirani 1999), nas revisões de Simaba e Simarouba (Cronquist 1944a,b), no estudo das espécies sul-americanas de Simaba (Cavalcante 1983), em trabalhos da série Flora do Paraguai (Muñoz 1990, Pirani 1987), da série Flora Ilustrada de Santa Catarina (Cowan \& Smith 1973, Fleig 1989, Pirani 1997) e da série Flora Fanerogâmica do Estado de São Paulo (Pirani 2002 a,b; Silva-Luz \& Pirani no prelo), e nos dados da Flora do Brasil (Daly \& Perdiz 2017, Pirani et al. 2017a,b, Silva-Luz \& Pirani 2017, Flores 2017). Os dados baseiam-se ainda em trabalho de campo no Mato Grosso do Sul e consultas aos acervos dos herbários CGMS, F, HRB, K, MBM, NY, R, RB, SP, SPF, UB e UEC.

\section{RESULTADOS E DISCUSSÃO}

Arepresentatividade de Picramniales e Sapindales (quadro 1) na flora do Mato Grosso do Sul é relativamente baixa: ambos são grupos muito mais ricos em espécies na Mata Atlântica e Amazônia. Gêneros inteiros de Burseraceae, Meliaceae, Rutaceae e Simaroubaceae são restritos a uma ou ambas dessas duas áreas florestais úmidas, sem qualquer indício de ocorrência no Brasil Central nem no MS. Porém existem espécies arbustivas e arbóreas de pequeno porte de Sapindales típicas dos cerrados, como Hortia oreadica, Spiranthera odoratissima (quadro 6), Anacardium humile e A. occidentale (quadro 3), ocorrendo com boa expressividade no MS.

Zanthoxylum rhoifolium (quadro 6), uma árvore aculeada muito comum, Astronium fraxinifolium, A. graveolens, Lithraea molleoides, Myracrodruon urundeuva e Tapirira guianensis (quadro 3) são amplamente distribuídas em todo MS, ocorrendo em várias formações vegetacionais, incluindo formações secundárias, em contraste com outras espécies restritas a habitats específicos ou a territórios pouco extensos, como é o caso das que existem apenas junto à fronteira com Paraguai e Bolívia, como Helietta puberula e Zanthoxylum rigidum (quadro 6), e das que alcançam seu limite máximo de distribuição no MS, seja limite ocidental como Picramnia sellowii (quadro 2), ou limite sul como Zanthoxylum ekmanii, ou limite noroeste como Pilocarpus pennatifolius (quadro 6), Schinus lentiscifolia, S. longifolia e $S$. weinmanniifolia (quadro 3). A espécie mais amplamente distribuída de Simarouba (S. amara) não foi registrada no MS até o presente. Já Simaba (quadro 7) é um gênero bem mais diversificado nos cerrados do Brasil central, na Mata Atlântica oriental e na Amazônia, porém até agora pouco documentado no MS.

Quadro 1. Número de espécies estimadas e conhecidas das ordens Picramniales e Sapindales no Mato Grosso do Sul.

\begin{tabular}{|c|c|c|c|c|c|}
\hline Ordem & Família & $\begin{array}{c}\text { Número de espécies } \\
\text { estimadas }\end{array}$ & $\begin{array}{l}\text { Número de espécies } \\
\text { conhecidas }\end{array}$ & $\begin{array}{c}\text { Número de espécies } \\
\text { naturalizadas }\end{array}$ & Quadro \\
\hline \multirow[t]{3}{*}{ Picramniales } & Picramniaceae & 3 & 2 & \multirow{3}{*}{$1 *$} & 2 \\
\hline & Anacardiaceae & 17 & 15 & & 3 \\
\hline & Burseraceae & $9 * *$ & 4 & & 4 \\
\hline \multirow[t]{3}{*}{ Sapindales } & Meliaceae & 18 & 15 & \multirow{3}{*}{$\begin{array}{c}1 * * * \\
3 * * * *\end{array}$} & 5 \\
\hline & Rutaceae & 26 & 22 & & 6 \\
\hline & Simaroubaceae & 8 & 5 & & 7 \\
\hline
\end{tabular}

Os asteriscos referem-se às informações sobre espécies e/ou híbridos naturalizados ou registros dúbios no estado do Mato Grosso do Sul. Tais informações estão inseridas no rodapé dos quadros $3,4,5$ e 6.

Quadro 2. Espécies da família Picramniaceae registradas com suas respectivas macrorregião e habitat no Mato Grosso do Sul.

\begin{tabular}{|c|c|c|}
\hline Espécie & Voucher & Macrorregião e habitat no MS \\
\hline Picramnia ramiflora Planch. & $\begin{array}{l}\text { Bodoquena, Serra da Bodoquena, Rodovia MS-178, } 40 \mathrm{~km} \mathrm{~S} \\
\text { de Bodoquena a Bonito, 22.11.2009, A. Pott } 15865 \text { (CGMS). }\end{array}$ & $\begin{array}{l}\text { Savana (Cerrado); áreas úmidas no interior de } \\
\text { mata semidecidual e de mata decidual. }\end{array}$ \\
\hline Picramnia sellowii Planch. & $\begin{array}{l}\text { Aquidauana, Serra de Maracaju, } 20^{\circ} 47^{\prime} 11^{\prime S} 55^{\circ} 78^{\prime} 72^{\prime \prime} \\
\text { W, G. Hatschbach et al. } 74932(\mathrm{MBM}, \mathrm{SPF}) \text {. }\end{array}$ & Savana (Cerrado); interior de mata ciliar. \\
\hline
\end{tabular}


Quadro 3. Espécies da família Anacardiaceae registradas com suas respectivas macrorregião e habitat no Mato Grosso do Sul.

\begin{tabular}{|c|c|c|}
\hline Espécie & Voucher & Macrorregião e habitat no MS \\
\hline Anacardium humile A. St.-Hil. & $\begin{array}{l}\text { Ponta Porã, cerca de } 21 \mathrm{~km} \text { de Ponta Porã em direção } \\
\text { à João Antônio, } 22^{\circ} 22^{\prime} \text { S, } 55^{\circ} 44^{\prime} \mathrm{W}, 10 . \mathrm{VIII} .2001, \text { V.C. } \\
\text { Souza et al. } 26770 \text { (CGMS, ESA, SPF). }\end{array}$ & Savana (Cerrado); cerrado. \\
\hline Anacardium occidentale L. & $\begin{array}{l}\text { Aquidauana, distrito de Piraputanga, próximo à } \\
\text { entrada da Associação dos Servidores da Junta do } \\
\text { Sossego, 5.VIII.2006, F.F Dias } 4 \text { (CGMS). }\end{array}$ & Savana (Cerrado); cerrado. \\
\hline Astronium fraxinifolium Schott & $\begin{array}{l}\text { Três Lagoas, em cerrado próximo a Ilha Solteira, } \\
\text { 1981, H.F. Leitão-Filho, } 12960 \text { (UEC). }\end{array}$ & $\begin{array}{l}\text { Savana (Cerrado), Mata Atlântica; cerrado, } \\
\text { mata decidual e mata semidecidual. }\end{array}$ \\
\hline Astronium graveolens Jacq. & $\begin{array}{l}\text { Campo Grande, BR } 262 \text { para Corumbá, ca. } 4 \text { km E do } \\
\text { trevo para Virgínia, 2001, J.R. Pirani et al. } 4830 \text { (SPF). }\end{array}$ & $\begin{array}{l}\text { Savana (Cerrado), Mata Atlântica; cerrado, mata } \\
\text { decidual, mata semidecidual e mata ciliar. }\end{array}$ \\
\hline Lithraea molleoides (Vell.) Engl. & $\begin{array}{l}\text { Maracaju, fazenda São João, } 12 \text { km NW Maracaju, } \\
21^{\circ} 31^{\prime} \mathrm{S}, 55^{\circ} 14^{\prime} \mathrm{W}, 5 . \mathrm{IX} .1994, \text { B. Dubs } 1728 \text { (CTES, } \\
\text { E, ESA, K, MBM, S, Z). }\end{array}$ & $\begin{array}{l}\text { Chaco, Savana (Cerrado) e Mata Atlântica; } \\
\text { cerrado, mata semidecidual e mata ciliar. }\end{array}$ \\
\hline Myracrodruon urundeuva Allemão & $\begin{array}{l}\text { Aquidauana, Piraputanga, 11.VIII.1970, G. } \\
\text { Hatschbach } 24579 \text { (MBM, NY). }\end{array}$ & $\begin{array}{l}\text { Savana (Cerrado), Mata Atlântica; cerrado, mata } \\
\text { decidual, mata semidecidual e mata ciliar. }\end{array}$ \\
\hline Schinopsis balansae Engl. & Porto Murtinho, XI.1940, (RB 46744). & Mata Atlântica; mata semidecidual. \\
\hline Schinopsis brasiliensis Engl. & $\begin{array}{l}\text { Corumbá, Piraputanga, encosta do sistema orográfico } \\
\text { denominado Maciço do Urucum, 30.V.1951, H.M. } \\
\text { Bastos s.n. (RB 73762). }\end{array}$ & Mata Atlântica; mata semidecidual. \\
\hline Schinus lentiscifolia Marchand & $\begin{array}{l}\text { Jardim, estrada não pavimentada para Guia Lopes, ramal } \\
\text { para rincão Bonito, a ca. } 3 \text { km da bifurcação, } 307 \text { m alt., } \\
\text { 26.I.2001, J.R. Pirani } 4873 \text { (FUEL, NY, SPF, UB). }\end{array}$ & Chaco e Savana (Cerrado); cerrado. \\
\hline Schinus longifolia (Lindl.) Speg. & Rio Brilhante, 27.I.1971, G. Hatschbach 26178 (NY). & Chaco e Savana (Cerrado); cerrado. \\
\hline Schinus molle L. & $\begin{array}{l}\text { Bela Vista, rodovia Bela Vista a Caracol, km 5-10, } \\
\text { 13.III.2004, G. Hatschbach et al. } 76979 \text { (MBM, RB). }\end{array}$ & Chaco e Savana (Cerrado); cerrado. \\
\hline Schinus terebinthifolia Raddi & $\begin{array}{l}\text { Campo Grande, Reserva Biológica - UFMS, } \\
\text { 17.VI.1995, R.N. Rodrigues s.n. (CGMS 5212). }\end{array}$ & $\begin{array}{l}\text { Chaco, Savana (Cerrado) e Mata Atlântica; } \\
\text { mata semidecidual e mata ciliar. }\end{array}$ \\
\hline Spondias mombin L. & $\begin{array}{l}\text { Corumbá, Reserva Indígena Guató, 26.XI.2001, G.A. } \\
\text { Damasceno-Junior } 3119 \text { (CGMS). }\end{array}$ & $\begin{array}{l}\text { Savana (Cerrado) e Mata Atlântica; mata } \\
\text { semidecidual e mata ciliar. }\end{array}$ \\
\hline Tapirira guianensis Aubl. & $\begin{array}{l}\text { Bodoquena, Serra da Bodoquena, em beira de córrego } \\
\text { sobre terreno calcário, no meio da vegetação de } \\
\text { cerrado, 1986, C.B.J. Jaramillo } 3738 \text { (SPF). }\end{array}$ & $\begin{array}{l}\text { Savana (Cerrado) e Mata Atlântica; cerrado, } \\
\text { mata semidecidual e mata ciliar. }\end{array}$ \\
\hline
\end{tabular}

*Mangifera indica L., originária da Ásia, conhecida popularmente como manga, encontra-se naturalizada em quase todos os Estados brasileiros, incluindo o MS.

Quadro 4. Espécies da família Burseraceae registradas com suas respectivas macrorregião e habitat no Mato Grosso do Sul.

\begin{tabular}{|c|c|c|}
\hline Espécie & Voucher & Macrorregião e habitat no MS \\
\hline Commiphora leptophloeos (Mart.) J.B.Gillett & Corumbá, J.R. Pirani et al. 4836 (SPF). & Chaco e Savana (Cerrado); mata decidual. \\
\hline Protium heptaphyllum (Aubl.) Marchand & $\begin{array}{l}\text { Bataiporã, } 22^{\circ} 29^{\prime} 53^{\prime \prime} \mathrm{S}, 53^{\circ} 27^{\prime} 11^{\prime \prime} \mathrm{W}, 1986 \text {, } \\
\text { U. Pastore } 152 \text { (MBM). }\end{array}$ & $\begin{array}{l}\text { Savana (Cerrado) e Mata Atlântica; cerrado, } \\
\text { mata ciliar e mata semidecidual. }\end{array}$ \\
\hline Protium ovatum Engl. & $\begin{array}{l}\text { Aquidauana, Camisão, 24.VII.2008, W.G. } \\
\text { Barbosa-Filho } 52 \text { (CGMS). }\end{array}$ & Savana (Cerrado); cerrado. \\
\hline Protium spruceanum (Benth.) Engl. & $\begin{array}{l}\text { Campo Grande, APA do Guariroba, } \\
\text { 18.VII.2008, M.L. Bueno } 600 \text { (CGMS). }\end{array}$ & $\begin{array}{l}\text { Savana (Cerrado); mata ciliar (espécie de } \\
\text { áreas alagáveis). }\end{array}$ \\
\hline
\end{tabular}

**Na Lista de Espécies do Brasil (Daly 2012) cinco outras espécies são também citadas para o MS: Crepidospermum rhoifolium (Benth.) Triana \& Planch., Protium grandifolium Engl., P. paniculatum Engl., P. polybotryum (Turcz.) Engl. e Trattinnickia rhoifolia Willd. Porém não encontramos registros dessas espécies nos herbários consultados nem no SpeciesLink, por isso sua ocorrência no MS não está confirmada. 
Quadro 5. Espécies da família Meliaceae registradas com suas respectivas macrorregião e habitat no mato Grosso do Sul.

\begin{tabular}{|c|c|c|}
\hline Espécie & Voucher & Macrorregião e habitat no MS \\
\hline Cabralea canjerana (Vell.) Mart. & $\begin{array}{l}\text { Caarapós, Fazenda Rancho Verde, 23.I.2001, } \\
\text { A. Sciamarelli } 842 \text { (CGMS). }\end{array}$ & $\begin{array}{l}\text { Mata Atlântica e Savana (Cerrado); mata } \\
\text { semidecidual e mata ciliar. }\end{array}$ \\
\hline Cedrela fissilis Vell. & $\begin{array}{l}\text { Ribas do Rio Pardo, córrego Campo Alegre, } \\
\text { 11.XI.1981, } 21^{\circ} 25^{\prime} \text { S } 53^{\circ} 54^{\prime} \mathrm{W}, \text { L.A. Dambrós } \\
246 \text { (RB). }\end{array}$ & $\begin{array}{l}\text { Mata Atlântica e Savana (Cerrado); mata } \\
\text { semidecidual e mata ciliar. }\end{array}$ \\
\hline Cedrela odorata L. & $\begin{array}{l}\text { Nioaque, Rodovia } 347,10 \mathrm{~km} \text { E da rodovia } \\
\text { BR-420, 19.IX.2007, A. Pott } 14543 \text { (CGMS). }\end{array}$ & $\begin{array}{l}\text { Mata Atlântica, Pantanal e Savana (Cerrado); } \\
\text { mata ciliar e matas alagáveis. }\end{array}$ \\
\hline Guarea guidonia (L.) Sleum. & $\begin{array}{l}\text { Corumbá, Roteamento Rita Vieira, } \\
\text { 11.VI.1987, C.A. Conceição } 2116 \text { (CGMS). }\end{array}$ & $\begin{array}{l}\text { Mata Atlântica e Savana (Cerrado); mata } \\
\text { semidecidual e mata ciliar. }\end{array}$ \\
\hline Guarea kunthiana A.Juss. & $\begin{array}{l}\text { Bonito, Rancho Tucano, Rio Formoso, } \\
21^{\circ} 10^{\prime} 16^{\prime} \mathrm{S}, 56^{\circ} 38^{\prime} 11^{\prime \prime} \mathrm{W}, 5 . \mathrm{IX} .1998, \text { G.A. } \\
\text { Damasceno-Junior } 1633 \text { (CGMS). }\end{array}$ & $\begin{array}{l}\text { Chaco e Savana (Cerrado); mata decidual e } \\
\text { mata semidecidual. }\end{array}$ \\
\hline Guarea macrophylla Vahl & $\begin{array}{l}\text { Campo Grande, Fazenda Santa Inês, } \\
\text { 4.VIII.1997, U.M. Resende } 1082 \text { (CGMS). }\end{array}$ & $\begin{array}{l}\text { Mata Atlântica e Savana (Cerrado); mata } \\
\text { semidecidual e mata ciliar. }\end{array}$ \\
\hline Trichilia catigua A.Juss. & $\begin{array}{l}\text { Bela Vista, } 22^{\circ} 10^{\prime} 899^{\prime S}, 56^{\circ} 52^{\prime} 11^{\prime \prime}, 1985 \text {, } \\
\text { G. Hatschbach } 49198 \text { (MBM, MO). }\end{array}$ & $\begin{array}{l}\text { Mata Atlântica e Savana (Cerrado); mata } \\
\text { semidecidual e mata ciliar. }\end{array}$ \\
\hline Trichilia claussenii C.DC. & $\begin{array}{l}\text { Bonito, } 21^{\circ} 12^{\prime} 11^{\prime \prime}, 56^{\circ} 48^{\prime} 19^{\prime} \mathrm{W}, 2002, G \text {. } \\
\text { Hatschbach } 74126 \text { (MBM). }\end{array}$ & $\begin{array}{l}\text { Chaco, Mata Atlântica e Savana (Cerrado); } \\
\text { mata decidual e mata emidecidual. }\end{array}$ \\
\hline Trichilia elegans A.Juss. & $\begin{array}{l}\text { Porto Murtinho, 2002, G. Hatschbach } 73991 \\
\text { (MBM). }\end{array}$ & $\begin{array}{l}\text { Mata Atlântica e Savana (Cerrado); mata } \\
\text { semidecidual e mata ciliar. }\end{array}$ \\
\hline Trichilia hirta L. & $\begin{array}{l}\text { Nioaque, Assentamento Andalucia, } \\
\text { 6.VII.2008, L.C.S. Magalhães } 378 \text { (CGMS). }\end{array}$ & $\begin{array}{l}\text { Chaco e Savana (Cerrado); mata decidual e } \\
\text { mata semidecidual. }\end{array}$ \\
\hline Trichilia pallida Sw. & $\begin{array}{l}\text { Campo Grande, Parque estadual do Prosa, } \\
20^{\circ} 44^{\prime} 27^{\prime} \text { S, 5464'63'W, 2.III.2003, L.C. } \\
\text { Rodrigues } 101 \text { (CGMS). }\end{array}$ & $\begin{array}{l}\text { Savana (Cerrado); mata semidecidual e mata } \\
\text { ciliar. }\end{array}$ \\
\hline Trichilia rubra C.DC. & $\begin{array}{l}\text { Corumbá, Fazenda Acurizal, 2.XII.1987, } A \text {. } \\
\text { Pott } 4017 \text { (CGMS, RB). }\end{array}$ & $\begin{array}{l}\text { Chaco e Savana (Cerrado); } \\
\text { mata semidecidual e mata ciliar. }\end{array}$ \\
\hline Trichilia silvatica C.DC. & $\begin{array}{l}\text { Jardim, Rio da Prata, 22.III.2003, J.L. } \\
\text { Battilani } 33 \text { (CGMS). }\end{array}$ & $\begin{array}{l}\text { Mata Atlântica e Savana (Cerrado); mata } \\
\text { semidecidual e mata ciliar. }\end{array}$ \\
\hline Trichilia schomburgkii C.DC. & $\begin{array}{l}\text { Corumbá, Fazenda Acurizal, Rio Miranda, } \\
\text { 24.II.1988, V. Pott } 471 \text { (CGMS, RB). }\end{array}$ & $\begin{array}{l}\text { Chaco e Savana (Cerrado); } \\
\text { mata semidecidual e mata ciliar. }\end{array}$ \\
\hline Trichilia stellatotomentosa Kuntze & $\begin{array}{l}\text { Corumbá, Morro Dourados, } 18^{\circ} 05^{\prime} 06^{\prime} \mathrm{S}, \\
57^{\circ} 49^{`} 15^{`} \mathrm{~W}, \text { G.A. Damasceno-Junior } 2552 \\
\text { (CGMS). }\end{array}$ & $\begin{array}{l}\text { Chaco e Savana (Cerrado); } \\
\text { mata decidual e mata semidecidual. }\end{array}$ \\
\hline
\end{tabular}

***Melia azedarach L., originária da Ásia e conhecida como cinamomo ou flor-de-Santa Bárbara, encontra-se naturalizada em várias partes do sul e oeste do Brasil, incluindo o MS.

Quadro 6. Espécies da família Rutaceae registradas com suas respectivas macrorregião e habitat no Mato Grosso do Sul.

\begin{tabular}{|c|c|c|}
\hline Espécie & Voucher & Macrorregião e habitat no MS \\
\hline Balfourodendron riedelianum (Engl.) Engl. & $\begin{array}{l}\text { Miranda, Serra da Bodoquena, } 20^{\circ} 24^{\prime} 06^{` S} \\
56^{\circ} 37^{\prime} 83^{\prime \prime} \mathrm{W}, \mathrm{G} . \text { Hatschbach et al. } 76342 \\
\text { (MBM, SPF). }\end{array}$ & $\begin{array}{l}\text { Mata Atlântica e Savana (Cerrado); mata } \\
\text { semidecidual. }\end{array}$ \\
\hline Ertela trifolia (L.) Kuntze & $\begin{array}{l}\text { Bodoquena, estrada para Bonito, ca. } 18 \mathrm{~km} \\
\text { S de Bodoquena, } 20^{\circ} 68^{\prime} 07^{\prime} \mathrm{S}, 56^{\circ} 63^{\prime} 08^{\prime \prime} \mathrm{W} \text {, } \\
\text { 2001, Pirani et al. } 4859 \text { (CGMS, SPF). }\end{array}$ & Savana (Cerrado); mata semidecidual. \\
\hline Esenbeckia almawillia Kaastra & $\begin{array}{l}\text { Ladário, Estrada Parque, } 19^{\circ} 11^{\prime} 27^{\prime} ’ \mathrm{~S} \text {, } \\
57^{\circ} 52^{\prime} 52^{\prime \prime W}, 2006, \text { P. Dias \& R.G. Udulutsch } \\
281 \text { (RB, SPF, UB). }\end{array}$ & Savana (Cerrado); cerrado. \\
\hline $\begin{array}{l}\text { Esenbeckia febrifuga (A.St.-Hil.) A.Juss. ex } \\
\text { Mart. }\end{array}$ & Iguatemi, G. Hatschbach 48401 (MBM, MO). & $\begin{array}{l}\text { Savana (Cerrado) e Mata Atlântica; mata } \\
\text { semidecidual. }\end{array}$ \\
\hline Esenbeckia grandiflora Mart. & $\begin{array}{l}\text { Aquidauana, Piraputanga, 19.II.2008, W. M. } \\
\text { Ramos } 141 \text { (CGMS). }\end{array}$ & $\begin{array}{l}\text { Savana (Cerrado) e Mata Atlântica; mata } \\
\text { ciliar e mata semidecidual. }\end{array}$ \\
\hline Esenbeckia hieronymi Engl. & Col. anônimo (R 71068) & Mata Atlântica; mata semidecidual. \\
\hline Esenbeckia leiocarpa Engl. & Bonito, V.R.B. Maria s.n. (ESA 51879) & Mata Atlântica; mata semidecidual. \\
\hline Galipea ciliata Taub. & $\begin{array}{l}\text { Ladário, Fazenda Vale do Paraíso, Morro Santa } \\
\text { Cruz, 2001, G. Damasceno et al. } 2162 \text { (CGMS). }\end{array}$ & Chaco; mata decidual. \\
\hline
\end{tabular}


Quadro 6. Cont.

\begin{tabular}{|c|c|c|}
\hline Espécie & Voucher & Macrorregião e habitat no MS \\
\hline Helietta apiculata Benth. & $\begin{array}{l}\text { Bela Vista, estrada para Antonio João, J. R. } \\
\text { Pirani et al. } 4879 \text { (NY, SPF). }\end{array}$ & Savana (Cerrado); mata semidecidual. \\
\hline Helietta puberula R.E.Fries & $\begin{array}{l}\text { Corumbá, J.R. Pirani et al. } 4844 \text { (MBM, NY, } \\
\text { SPF). }\end{array}$ & Chaco; mata decidual. \\
\hline Hortia oreadica Groppo, Pirani \& Kallunki & $\begin{array}{l}\text { Campo Grande, 15.VII.1997, U.M. Resende } \\
1386 \text { (CGMS, SPF). }\end{array}$ & Savana (Cerrado); cerrado. \\
\hline Pilocarpus pennatifolius Lem. & $\begin{array}{l}\text { Antônio João, descida para Campestre, 1985, } \\
\text { G. Hatschbach \& F.J. Zelma } 49111 \text { (MBM, } \\
\text { MO, UPCB). }\end{array}$ & Mata Atlântica; mata semidecidual. \\
\hline Spiranthera odoratissima A.St.-Hil. & Rio Verde, 1979, P.I. Oliveira 27 (MBM, MO). & Savana (Cerrado) e Chaco; cerrado. \\
\hline Zanthoxylum acuminatum (Sw.) Sw. & $\begin{array}{l}\text { Bodoquena, estrada para Bonito, 2001, J.R. } \\
\text { Pirani et al. } 4858 \text { (SPF). }\end{array}$ & $\begin{array}{l}\text { Savana (Cerrado) e Mata Atlântica; mata } \\
\text { semidecidual. }\end{array}$ \\
\hline Zanthoxylum caribaeum Lam. & $\begin{array}{l}\text { Miranda, Retiro da Fazenda Xaraés, } \\
\text { 15.VII.1990, C.A. Conceição } 2643 \text { (CGMS). }\end{array}$ & $\begin{array}{l}\text { Savana (Cerrado) e Chaco; mata semidecidual } \\
\text { e mata decidual. }\end{array}$ \\
\hline Zanthoxylum ekmanii (Urb.) Alain & $\begin{array}{l}\text { Bodoquena, fazenda Califórnia, 22.V.2002, S. } \\
\text { Aragaki } 942 \text { (CGMS). }\end{array}$ & Chaco e Savana; mata semidecidual. \\
\hline Zanthoxylum fagara (L.) Sarg. & $\begin{array}{l}\text { Corumbá, Nabileque, Pantanal, 1987, A. Pott } \\
\text { et al. } 4055 \text { (CGMS, SPF). }\end{array}$ & $\begin{array}{l}\text { Pantanal, Chaco e Savana; mata decidual e } \\
\text { mata semidecidual. }\end{array}$ \\
\hline Zanthoxylum petiolare A.St.-Hil. \& Tul. & $\begin{array}{l}\text { Corumbá, Nhecolândia, C.N. Cunha et al. } \\
2093 \text { (CGMS, SPF). }\end{array}$ & Pantanal e Savana; mata semidecidual. \\
\hline Zanthoxylum rhoifolium Lam. & $\begin{array}{l}\text { Bonito, 1998, O.S. Ribas et al. } 2381 \text { (MBM, } \\
\text { SPF) }\end{array}$ & $\begin{array}{l}\text { Pantanal, Savana e Mata Atlântica; mata } \\
\text { semidecidual, mata ciliar, cerrado, vegetação } \\
\text { pioneira (áreas perturbadas). }\end{array}$ \\
\hline Zanthoxylum riedelianum Engl. & $\begin{array}{l}\text { Corumbá, Fazenda Santana, 6.VII.1985, } A . \\
\text { Pott } 1994 \text { (CGMS). }\end{array}$ & $\begin{array}{l}\text { Savana; cerrado, cerradão e mata } \\
\text { semidecidual. }\end{array}$ \\
\hline Zanthoxylum rigidum Kunth ex Willd. & $\begin{array}{l}\text { Corumbá, 2001, D.A. Damasceno Jr. et al. } \\
3098 \text { (CGMS) }\end{array}$ & $\begin{array}{l}\text { Chaco, Savana, Pantanal; mata ciliar, cerrado, } \\
\text { murundus em campo inundável. }\end{array}$ \\
\hline Zanthoxylum sprucei Engl. & Bonito, 1998, V.A. Pott 3834 (CGMS) & Pantanal e Savana, mata semidecidual. \\
\hline
\end{tabular}

**** Os seguintes táxons cítricos, nativos da Ásia e introduzidos no Brasil há séculos, encontram-se naturalizados em várias partes do sul e oeste do Brasil e Paraguai: Citrus x aurantium L. (laranjeira), C. x limon (L.) Osbeck (limoeiro, limão-cravo) e C. reticulata Blanco (mixiriqueira) (e.g. Gade 1976, Pirani et al. 2017a).

Quadro 7. Espécies da família Simaroubaceae registradas com suas respectivas macrorregião e habitat no Mato Grosso do Sul.

\begin{tabular}{|c|c|c|}
\hline Espécie & Voucher & Macrorregião e habitat no MS \\
\hline Simaba glabra Engl. & $\begin{array}{l}\text { Bonito, estrada para Guia Lopes, } \\
21^{\circ} 10^{\prime} 15.8^{\prime} \mathrm{S}, 56^{\circ} 24^{\prime} 38.6 ” \mathrm{~W}, \mathrm{I} .2001, \text { J.R. } \\
\text { Pirani et al. } 4872 \text { (SPF). }\end{array}$ & Savana (Cerrado); cerrado. \\
\hline Simaba intermedia Mansf. & $\begin{array}{l}\text { Aquidauana, Cipolândia, 29.IX.2007, A. Pott } \\
\& \text { V. Pott } 14555 \text { (CGMS, SPF). }\end{array}$ & Savana (Cerrado); cerrado e mata ciliar. \\
\hline Simaba salubris Engl. & Campo Grande, 1936, Archer \& Gehrt 95 (SP). & Savana (Cerrado); cerrado. \\
\hline Simaba trichilioides A.St.-Hil. & $\begin{array}{l}\text { Sonora, Reserva Legal da usina (CASE), } \\
\text { 5.VIII.2001, U.M. Resende } 2664 \text { (CGMS). }\end{array}$ & Savana (Cerrado); cerrado. \\
\hline Simarouba versicolor A.St.-Hil. & $\begin{array}{l}\text { Três Lagoas, } 9,4 \text { km da MS-395, 2048'57’S, } \\
51^{\circ} 43 \text { '26”W, 13.X.1996, A. Amaral et al. } 75 \\
\text { (BOTU, SPF). }\end{array}$ & Savana (Cerrado); cerrado e mata ciliar. \\
\hline
\end{tabular}

\section{Perspectivas de pesquisa sobre o grupo nos próximos 10 anos}

O interesse pelas plantas das duas ordens tratadas aqui tem se mostrado crescente. Trata-se de plantas importantes pela frequência na natureza, pelo aproveitamento econômico, pela variabilidade morfológica e necessidade de novos estudos suscitados pelas filogenias recentes. A existência atual de especialistas em instituições de botânica relevantes e crescente número de discípulos sendo formados evidenciam boa perspectiva de incremento dos estudos e aprofundamento das abordagens sobre o grupo. Também a maior exploração no âmbito ecológico, com vistas ao mapeamento da vegetação, deve auxiliar no preenchimento das lacunas supracitadas. A disponibilidade e uso desses dados mais acurados deverá permitir mais consistência nas ações visando à conservação biológica das espécies desses grupos no Mato Grosso do Sul. 


\section{AGRADECIMENTOS}

Os autores agradecem aos curadores dos herbários citados no texto pelo apoio; aos colegas nas expedições a campo pela colaboração; ao Conselho Nacional de Desenvolvimento Científico e Tecnológico e à Fundação de Amparo à Pesquisa do Estado de São Paulo pelo apoio aos projetos de pesquisa coordenados pelo primeiro autor.

\section{REFERÊNCIAS}

Angiosperm Phylogeny Group - APG III. 2009. An update of the Angiosperm Phylogeny Group classification for the orders and families of flowering plants: APG III. Botanical Journal of the Linnean Society 161:105-121.

Barkley, F.A. 1957. A study of Schinus L. Lilloa 28:5-110. 365 . 1962. Anacardiaceae: Rhoideae: Lithraea. Phytologia 8(7):329-

1968. Anacardiaceae: Rhoideae: Astronium. Phytologia 16(2):107-152.

Cavalcante, P.B. 1983. Revisão taxonômica do gênero Simaba Aubl. (Simaroubaceae) na América do Sul. Publicações Avulsas do Museu Goeldi 37:1-85.

Chase, M.W. \& Reveal, J.L. 2009. A phylogenetic classification of the land plants to accompany APG III. Botanical Journal of the Linnean Society 161:122-127.

Consulta ao catálogo da Rede Brasileira de Herbários. Disponível em: www.botanica.org.br-rede_herbarios. Acessado em 23.11.2016.

Cowan, R.S. \& Smith, L.B. 1973. Rutáceas. In Flora Ilustrada Catarinense (R. Reitz, ed.) Itajaí, Herbário Barbosa Rodrigues. 89 p.

Cronquist, A. 1944a. Studies in the Simaroubaceae. III. The genus Simaba. Lloydia 7(1):81-92.

1944b. Studies in the Simaroubaceae. III. The genus Simarouba. Bullettin of the Torrey Botanical Club 71(3):226-234.

Daly, D.C. 2012. Burseraceae In Lista de Espécies da Flora do Brasil. Jardim Botânico do Rio de Janeiro. Disponível em: http://floradobrasil. jbrj.gov.br/2012/FB016657. Acessado em 15.11.2012.

Daly, D.C. \& Perdiz, R.O. 2017. Burseraceae. In Flora do Brasil 2020 em construção. Jardim Botânico do Rio de Janeiro. Disponível em: http://floradobrasil.jbrj.gov.br/reflora/floradobrasil/FB44. Acessado em 27.11.2017

Dubs, B. 1998. Prodromus Florae Matogrossensis. Betrona, Küsnacht, $444 \mathrm{p}$.

Engler, A. 1874a. Rutaceae. In Flora brasiliensis (C.F.P. Martius \& A.G. Eichler, eds.) Frid. Fleischer, Lipsiae, vol. 12, pars 2, p. 75-196, tabs. 14-39.

1874b. Simaroubaceae. In Flora brasiliensis (C.F.P. Martius \& A.G. Eichler,eds.) Frid. Fleischer, Lipsiae, vol. 12, pars 2, p. 197-248, tabs. 40-49.

1876. Anacardiaceae. In Flora brasiliensis (C.F.P..Martius \& A.G. Eichler, eds.) Frid. Fleischer, Lipsiae, vol.12, pars 2, p. 367-418.

Fleig, M. 1989. Anacardiáceas. In Flora Ilustrada Catarinense (R. Reitz, ed.) Herbário Barbosa Rodrigues, Itajaí. 64 p.

Flores, T.B. 2017. Meliaceae. In Flora do Brasil 2020 em construção. Jardim Botânico do Rio de Janeiro. Disponível em: http://floradobrasil. jbrj.gov.br/reflora/floradobrasil/FB44. Acessado em 27.11.2017
Gade, D.W. 1976. Naturalization of plant alliens: the volunteer orange in Paraguay. Journal of Biogeography 3:269-279.

Groppo, M. \& Pirani, J.R. 2012. A revision of Hortia (Rutaceae). Systematic Botany 37(1):197-212.

Kaastra, R.C. 1982. Pilocarpinae (Rutaceae). Flora Neotropica 33:1-198.

Muñoz, J.D. 1990. Anacardiaceae. In Flora del Paraguay. Conservatoire et Jardin botaniques de la Ville de Géneve. Missouri Botanical Garden, Saint Louis. 84 p.

Pennington, T.D. 1981. Meliaceae. Flora Neotropica 28:1-470.

Pennington, T.D. \& Muellner, A.N. 2010. A monograph of Cedrela (Meliaceae). Dh books, Milborne Port. $112 \mathrm{p}$.

Pirani, J.R. 1990. As espécies de Picramnia Sw. (Simaroubaceae) do Brasil: uma sinopse. Boletim de Botânica da Universidade de São Paulo 12:115-180.

1998. A revision of Helietta and Balfourodendron (RutaceaePteleinae). Brittonia 50(3):348-380.

1999 (inédito). Estudos taxonômicos em Rutaceae do Brasil. Tese. 197 p. Universidade de São Paulo.

1987. Simaroubaceae. In Flora del Paraguay. Genève, Conservatoire et jardin Botaniques de la Ville de Genève \& SaintLouis (R. Spichiger, ed.). Missouri Botanical Garden. 28 p.

1997. Simaroubaceae. In Flora Ilustrada Catarinense (A. Reis, ed.). Herbário Barbosa Rodrigues, Itajaí. 48 p.

2002a. Rutaceae. In Flora Fanerogâmica do Estado de São Paulo (M.G.L. Wanderley, G.J. Shepherd \& A.M. Giulietti, eds.) São Paulo, Fundação de Amparo à Pesquisa do Estado de São Paulo, HUCITEC, vol. 2, p. 281-308.

2002b. Simaroubaceae. In Flora Fanerogâmica do Estado de São Paulo (M.G.L. Wanderley, G.J. Shepherd \& A.M. Giulietti, eds.) São Paulo, Fundação de Amparo à Pesquisa do Estado de São Paulo, HUCITEC, vol. 2, p. 313-321.

2012. Picramniaceae. In Lista de Espécies da Flora do Brasil. Jardim Botânico do Rio de Janeiro. Disponível em: http:// floradobrasil.jbrj.gov.br/2012/FB000188. Acessado em 15.11.2012.

Pirani, J.R., Groppo, M. \& Dias, P. 2017a. Rutaceae. In Flora do Brasil 2020 em construção. Jardim Botânico do Rio de Janeiro. Disponível em: http://floradobrasil.jbrj.gov.br/reflora/floradobrasil/FB44. Acessado em 27.11.2017

Pirani, J.R., Devecchi, M.F. \& Thomas, W. 2017b. Simaroubaceae. In Flora do Brasil 2020 em construção. Jardim Botânico do Rio de Janeiro. Disponível em: http://floradobrasil.jbrj.gov.br/reflora/floradobrasil/ FB44. Acessado em 27.11.2017

Santin, D.A. \& Leitão-Filho, H.D. 1991. Restabelecimento e revisão taxonômica do gênero Myracrodruon Freire Allemão (Anacardiaceae). Revista Brasileira de Botânica 14:133-145.

Silva-Luz, C.L. \& Pirani, J.R. 2012. Anacardiaceae. In Lista de Espécies da Flora do Brasil. Jardim Botânico do Rio de Janeiro. Disponível em: http://floradobrasil.jbrj.gov.br/2012/FB000044. Acessado em 15.12.2012.

Silva-Luz, C.L. \& Pirani, J.R. 2017. Anacardiaceae. In Flora do Brasil 2020 em construção. Jardim Botânico do Rio de Janeiro. Disponível em: http://floradobrasil.jbrj.gov.br/reflora/floradobrasil/FB44. Acessado em 27.11.2017.

Silva-Luz, C.L. \& Pirani, J.R. (no prelo). Anacardiaceae. In Flora fanerogâmica do Estado de São Paulo. Instituto de Botânica, São Paulo. 